\title{
Forty Years of the First Attempt at the Electroweak Unification and of the Prediction of the Weak Neutral Boson $Z_{0}$
}

\author{
J. Leite Lopes \\ Centro Brasileiro de Pesquisas Físicas - CBPF \\ Rua Dr. Xavier Sigaud, 150 \\ 22090-180 - Rio de Janeiro, RJ - Brazil \\ Received 24 May, 1999
}

\begin{abstract}
The author describes his first attempt in 1958 at the unification of electromagnetic and weak interactions and his prediction in the same paper of the neutral $Z_{0}$ boson which would be the intermediate quantum exchanged in an eventual electron-neutron weak interaction (as muonic neutrinos were not known at that time).
\end{abstract}

\section{Introduction}

Enrico Fermi [1] was the first to give a theoretical description of the neutron beta-decay, which became the foundation of the theory of weak interactions. Historically, Fermi was also the first to propose an important application of the ideas of quantum electrodynamics which were developed mainly by P.A.M. Dirac [2], W. Heisen- berg and W. Pauli [3], P. Jordan and E.P. Wigner [4] and by Fermi himself [5]. In his article, Fermi says that according to the quantum theory of radiation, the number of photons in a system is not constant: photons are created when they are emitted and annihilated when they are absorbed. He, therefore, postulated in his theory of the neutron beta-decay that the "otal number of electrons as well as of the neutrinos, is not necessarily constant". Each transition from neutron to proton is associated with the creation of an electron and of a neutrino. The reverse process, however, the transformation of a proton into a neutron, is to be associated with the disappearence of an electron and of a neutrino. He then replaced the electromagnetic field $A_{\mu}(x)$ in the interaction lagrangean of this field with the electromagnetic current

$$
J^{\mu}(x)=\bar{\psi}(x) \gamma^{\mu} \psi(x)
$$

namely

$$
\mathcal{L}_{\gamma}=e\left(\bar{\psi}(x) \gamma^{\mu} \psi(x) A_{\mu}(x)\right)
$$

by a formula which describes the creation of an electron and an anti-neutrino - that is to say, $\bar{e}(x) \gamma^{\mu} \nu(x)$, and the electric current by one describing the transition neutron-proton. If $\mathcal{G} / \sqrt{2}$ is the constant which replaces the charge e and which expresses the intensity of the weak interactions, Fermi postulated the lagrangean of his beta-ray theory namely:

$$
L_{W}=\frac{\mathcal{G}}{\sqrt{2}}\left(\bar{p}(x) \gamma^{\mu} n(x)\right)\left(\bar{e}(x) \gamma_{\mu} \nu(x)\right)
$$

where we adopt the notation of the particle to indicate its spinor operator.

The analogy with electrodynamics incited him to choose a vector interaction. Several authors [6], just after Fermi's paper publication, besides studying other possible geometric forms of interaction, studied the possibility that the exchange of electron-antineutrino pairs between a neutron and a proton might give rise to a neutron-proton interaction, similar to the electromagnetic interaction between charged particles which results from virtual photon exchanges between the particles. This attempt was not successful and was followed by the introduction by Hideki Yukawa [7] of the idea of an intermediate massive boson exchanged between the nucleons and which would generate the nucleon interaction. The mass of this boson was determined by Yukawa by taking into account the range of the nuclear forces.

At that time there was a prejudice among physicists 
against the idea of new particles - Einstein's photon was accepted only after its evidence in the Compton effect - so Yukawa's idea was taken seriously only after the discovery of particles with Yukawa's boson mass in the cosmic radiation by S.H. Neddermeyer and C.D. Anderson [8]. It turned out later that Yukawa's bosons are the Lattes, Occhialini and Powell [9] pions with spin zero, whereas Anderson and Neddermeyer particles are rather muons, with spin $1 / 2$, leptons therefore [10].

Yukawa's intention that his theory would be able to describe both the strong interactions and the weak coupling did not meet with success in regard to the weak interactions [11].

The lack of knowledge of the precise form of the weak interactions was an obstacle to the consideration of intermediate bosons to induce these interactions would they be scalar, pseudoscalar, tensor or vector bosons?

It was only after the paper by R.P. Feynman and M. Gell-Mann [12] as well as those by E.G.C. Sudarshan and R.E. Marshak [13] and J. Sakurai [14], that the form of the weak interaction was established as a special combination of a vector current $V$ and an axialvector current $A$, namely $V-A$, in interaction with itself.

In their article, Feynman and Gell-Mann write:

"We have adopted the point of view that the weak interactions all arise from the interaction of a current $J_{\mu}$ with itself, possibly via an intermediate charged vector meson of high mass".

Therefore, the idea of intermediate vector bosons in Fermi's interaction became possible in spite of the difficulties of this model: indeed, as in the year 1958 the existence of muonic neutrinos was not known, G. Feinberg [15] showed that the absense of the radiative disintegration of the muon.

$$
\mu \rightarrow e+\gamma
$$

was imcompatible with the hypothesis of the intermediate vector-bosons. Indeed, with only one neutrino accompanying both electrons and muons this decay would be possible according to the diagram (and two other diagrams):

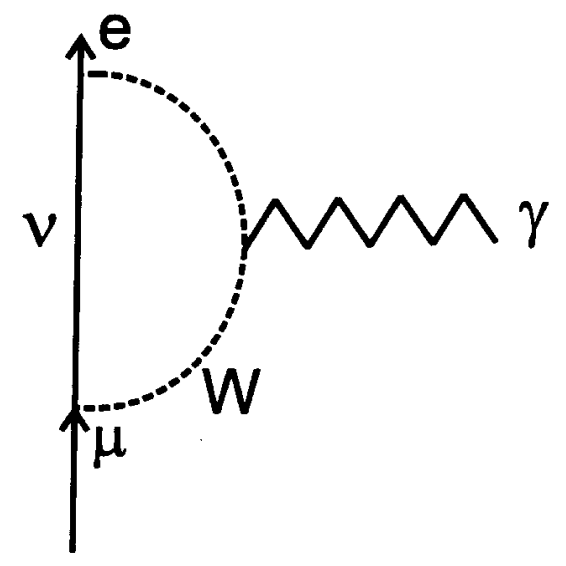

Figure 1

whereas with $\nu_{\mu} \neq \nu_{e}$ and a companion of only muons one could not have $\nu_{\mu}$ connected to the electron.

It was in the year 1958 that, as I read FeynmanGell-Mann paper, I had the immediate feeling that if weak interactions were due to the exchange of intermediate vector bosons they would have to be intimately related to the electromagnetic interactions transmitted by photons which are also vector particles.

An idea of unification of these interactions, I proposed it [16] in assuming that the intensity of the electromagnetic interactions $\underline{e}$ between electric particles and the electromagnetic field is equal to the intensity of the weak interactions, $\underline{g}$ between the weak currents and the boson field:

$$
e=g
$$

an idea which is implicit in this equality and in the same geometric nature of both photons and intermediate bosons $W$.

In fact, as an electric charge the constant $\underline{e}$ is universal for all observable charged particles (confined quarks have fractions of $\underline{e}$ as charge) so the above equation extends the universality of $\underline{e}$ as a coupling constant.

Now the amplitude for the reaction

$$
\bar{\mu} \rightarrow \nu_{\mu}+e+\bar{\nu}_{e}
$$

according to the Fermi point-like interaction 


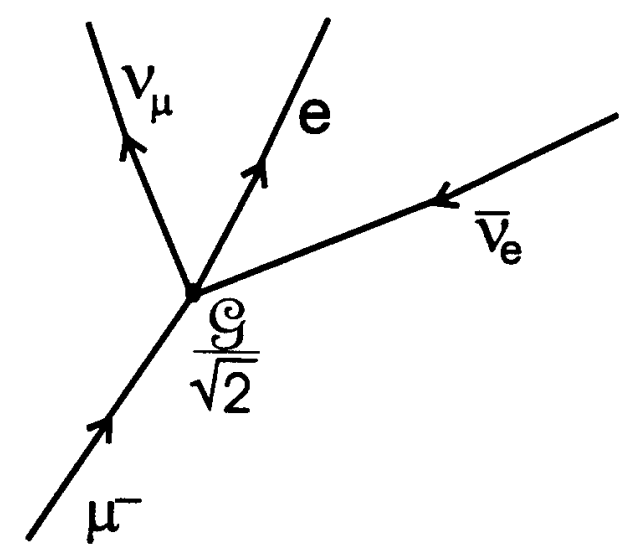

\section{Figure 2}

contains the expression

$\frac{\mathcal{G}}{\sqrt{2}}\left(\bar{\nu}_{\mu}\left(p_{\nu_{\mu}}\right) \gamma^{\alpha}\left(1-\gamma^{5}\right) \mu\left(p_{\mu}\right)\right)\left(\bar{e}\left(p_{e}\right) \gamma_{\alpha}\left(1-\gamma^{5}\right) \nu_{e}\left(p_{\nu_{e}}\right)\right)$

whereas the amplitude for this reaction via the intermediate bosons $W$ :

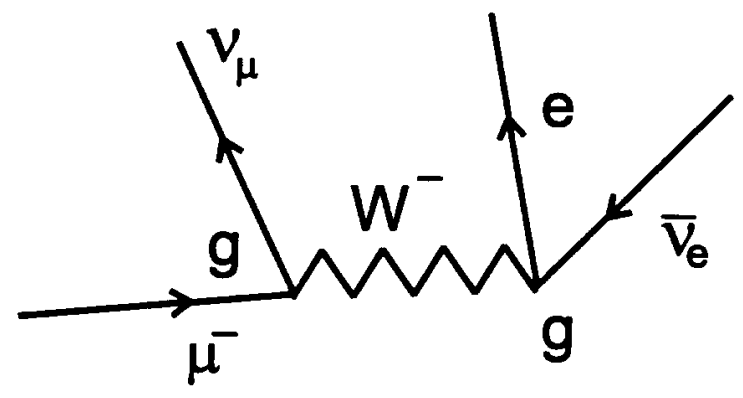

Figure 3

contains the formula

$$
\begin{gathered}
-g^{2}\left(\bar{\nu}_{\mu}\left(p_{\nu_{\mu}}\right) \gamma^{\alpha}\left(1-\gamma^{5}\right) \mu\left(p_{\mu}\right)\right)\left(\eta_{\alpha \beta}-\frac{k_{a} k_{\beta}}{m_{W}^{2}}\right) \\
\cdot \frac{1}{k^{2} m_{W}^{2}}\left(\bar{e}\left(p_{e}\right) \gamma^{\beta}\left(1-\gamma^{5}\right) \nu\left(p_{\nu_{e}}\right)\right)
\end{gathered}
$$

where

$$
k^{2}=p_{\nu_{\mu}}-p_{\mu}
$$

If the momentum transfer is very small with respect to the boson mass $m_{W}$ :

$$
k^{2}<<m_{W}^{2}
$$

then the two graphs will coincide, the amplitudes will be identical provided that:

$$
\frac{\mathcal{G}}{\sqrt{2}}=\frac{g^{2}}{m_{W}^{2}}
$$

a relation between the Fermi constant, experimentally known, and the unknown parameters, the mass $m_{W}$ and the coupling constant $g$.

It was here that I replaced $g$ by $\underline{e}$ according to equation (1) and this allowed me to evaluate $m_{W}$. I obtained $m_{W} \sim 40 m_{p}$. (in fact due to factors I included in this formula the value I indicated was $60 \mathrm{~m}_{p}$ ).

Once the idea of weak interactions mediated by vector bosons was taken seriously the question arose to me if there would not exist weak interactions due to an exchange of neutral vector bosons between neutral weak currents. I was influenced by the pion interaction with nucleons, the invariance of which under the group $\mathrm{SU}_{2}$ gives only one coupling constant for the nucleon current in interaction with the pion field. First proposed by N. Kemmer the charge-independent theory states that:

$$
\frac{1}{\sqrt{2}} f_{c}=f_{p}=-f_{n} \equiv f
$$

where $f_{c}$ is the coupling constant of charged pions with neutron-proton currents, $f_{p}$ and $f_{n}$ terms couple neutral pions with proto-proton and neutron-neutron currents respectively.

What would happen if we assumed neutral vectorbosons in weak interactions together with the charged vector bosons? I assumed wrongly that the exchange of neutral vector bosons would give a parity conserving interaction so as to have neutral current conserved; but I pointed out that the neutral vector boson-now baptised $Z_{0}$-would give a weak electron-neutron interaction so that the diagram

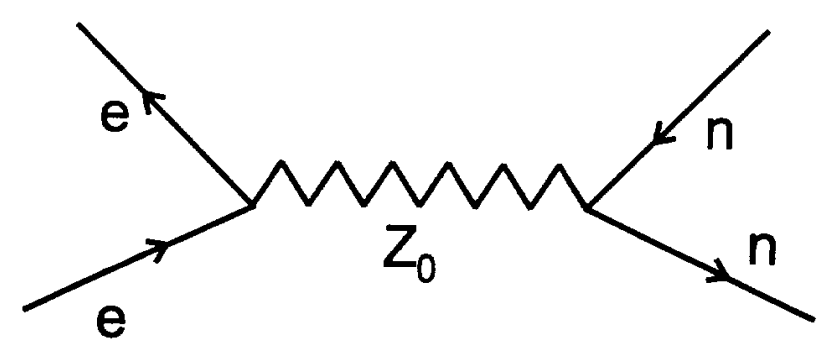

Figure 4

is predominant over the second order diagram: 


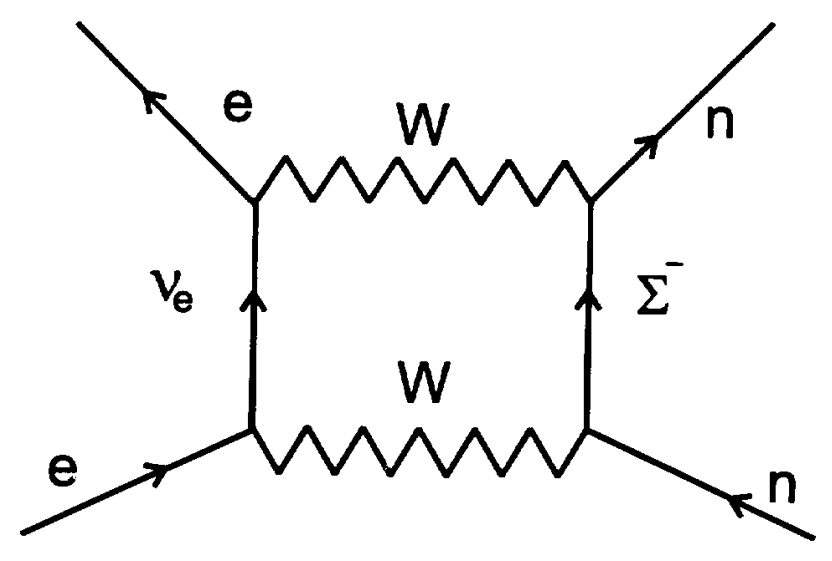

Figure 5

That is the experiment which occurred to me since in 1958 muonic neutrinos were not known and much less their beams.

I therefore proposed an alternative theory to that of Feymman-Gell-Mann:

I supposed the existence of neutral vector bosons together with the charged vector bosons. In fact they wrote in their paper: "We deliberately ignore the possibility of a neutral current, containing terms like $(\bar{e}-e)$, $(\bar{\mu} e),(\bar{n} n)$ etc and possibly coupled to a neutral intermediate field"1.

I thought that there was no reason to ignore possible neutral vector bosons as we knew that neutral pions were found only after charged pions were revealed.

My paper was thus the first to give a value for the mass of the $W$ bosons of the order of magnitude of their expperimental value. Two years later, T.D. Lee and C.N. Yang [17] indicated that mw should be larger than the mass of kaons in order to justify the absence of the radiative decay $K^{ \pm} \rightarrow W^{ \pm}+\gamma$. And according to B. Pontecorvo [18] "in 1959 the intermediate boson (without serious reasons) was supposed to have a mass of a few GeV".

As I communicated my results to Pontecorvo, he wrote me a letter in which he says to have inserted in the Russian version of his paper to the International Colloquium on the particle physics history in Paris (1982): "This question is still alive today, but nowadays we have the Glashow, Salam and Weinberg theory which predicts that the intermediate bosons masses are $\sim 100 \mathrm{GeV}$, whereas in 1959 only a few scientists,

\footnotetext{
${ }^{1}$ Ref.[12], pg. 197.
}

among them Ya Zeldovich and J. Leite Lopes, had the opinion that intermediate meson masses may be $\sim 100$ $G e V$, while it was generally believed (without serious reasons) that these masses are only a few GeV".

The value of the masses of $m w$ and the zero mass of photons inhibited me to say that they form a multiplet.

And my prediction of the $Z_{0}$ boson was not an academic exercise since I indicated that it would be the intermediate quantum in electron-neutron elastic scattering due to weak interactions. The preprint of my paper was read by Abdus Salam, according to Jayme Tiomno, who was at that time at the London Imperial College, and Salam told him that it contained good ideas. This remark was followed by several papers published by A. Salam and J. Ward [19] but I did not have the honour to be quoted by them. However, Steven Weinberg [20] quoted my paper and the paper by $\mathrm{S}$. Bludman [21] and C.N. Yang [22] as well as Tiomno [23] made a positive comment on this paper.

The neutral bosons $Z_{0}$ are, as well knwon, also predicted by the electroweak model and equation (1) is replaced by the relationship:

$$
e=g \sin \theta_{W}
$$

where the angle $\theta_{W}$ is the Weinberg angle which defines the proportion in which the gauge fields enter to define the electromagnetic field $A_{\mu}$ and the neutral boson field $Z_{\mu}$.

I was delighted in reading Weinberg's papers and in 1972 I [24] proposed that the unification of photons and $Z_{0}$ would enter the vector dominance model so that the vector bosons $\rho^{ \pm}$would also be related to the intermediate vector bosons $W^{ \pm}$, as $\rho^{0}$ is related to $\gamma$ and $Z_{0}$.

The model of Weinberg, Salam and Glashow gave the theoretical reasons for my intuitive inductions, and based on the Higgs mechanism, is the first example of the unification of physical forces.

\section{References}

[1] E. Fermi, Zs. f. Phys. 88, 161 (1934); F. Perrin, C.R. Acad. Sci Paris 197, 1625 (1933).

[2] P.A.M. Dirac, Proc. Roy. Soc. A, 114, 243, 710 (1927).

[3] W. Heisenberg and W. Pauli, Zs. f. Phys. 56, 1 (1929).

[4] P. Jordan and E.P. Wigner, Zs. f. Phys. 47, 631 (1928).

[5] E. Fermi, Rev. Mod. Phys. 4, 131 (1932). 
[6] D. Iwanenko, Nature (London 133, 981 (1934); Ig. Tamm, Nature (London 133, 982 (1934); G.C. Wick, Rend. Lincei 21, 170 (1935); M. Fierz, Zs. f. Phys. 104, 553 (1936).

[7] H. Yukawa, Proc. Phys. Math. Soc. Japan 17, 48 (1935).

[8] C.D. Anderson and S.H. Neddermeyer, Phys. Rev. 51, 884 (1937).

[9] C.M.G. Lattes, G.P.S. Occhialini and C.F. Powel, Nature (London) 16D, 453 (1947).

[10] B. Pontecorvo, Phys. Rev. 72, 246 (1947); O. Klein, Nature (London) 161, 897 (1948); J. Tiomno and J.A. Wheeler, Rev. Mod. Phys. Rev. Mod. Phys. 21, 144 (1944); 21, 153 (1949).

[11] See E. Amaldi, Colloque International sur l'histoire de la physique des particles, Journal de Physique, Supplement. $\mathrm{n}^{\circ}$ 12, Tome 43, pg. C8-261, Paris (1982).

[12] R.P. Feynman and M. Gell-Mann, Phys. Rev. 109, 193 (1958).

[13] E.C.G. Sudarsham and R.E. Marshak, Proc. Padua Conf. on mesons recently discov. part. p.v-14 (1957).
[14] J. Sakurai, Nuovo Cimento 7, 649 (1958).

[15] G. Feinberg, Phys. Rev. 110, 1482 (1958).

[16] J. Leite Lopes, Nucl. Phys. 8, 234 (1958).

[17] T.D. Lee and C.N. Yang, Phys. Rev. 119, 1410 (1960).

[18] B. Pontecorvo, Colloque Intern. Histoire Phys. Particle, Journal de Physique, Supplemt. $\mathrm{n}^{\mathrm{O}}$ 12, Tome, pg. C8-221, Paris (1982).

[19] A. Salam and J.C. Ward, Nuovo Cim. 11, 568 (1959).

[20] S. Weinberg, Phys. Rev. Letters 19, 1264 (1967).

[21] S. Bludman, Nuovo Cimento 9, 433 (1958).

[22] C.N. Yang, Selected papers 1945-1980 with commentary, pg. 47, W.H. Freeman and Co., San Francisco (1983).

[23] J. Tiomno, Colloque Intern. Histoire Phys. Particles, Journal de Physique, Supplem. $\mathrm{n}^{\mathrm{O}}$ 12, Tome 43, pg. C8-299, Paris (1982).

[24] J. Leite Lopes. Nucl. Phys. B38, 555 (1972). 